OPEN ACCESS

Edited by: Ilaria Marigo,

Veneto Institute of Oncology (IRCCS), Italy

Reviewed by: Matthias Bozza,

German Cancer Research Center (DKFZ), Germany

Jan Joseph Melenhorst, University of Pennsylvania, United States Aarthi Talla,

Allen Institute for Immunology, United States

*Correspondence: Nirjal Bhattara Nirjal.Bhattarai@FDA.HHS.Gov

${ }^{\dagger}$ Present address: Joseph W. Fischer, Oncology R\&D Division, Cell Therapy Group, AstraZeneca, Gaithersburg, $M D$, United States

Specialty section: This article was submitted to Cancer Immunity and Immunotherapy, a section of the journal Frontiers in Immunology

Received: 09 April 2021 Accepted: 04 June 2021

Published: 18 June 2021

Citation: Fischer JW and Bhattarai N (2021) CAR-T Cell Therapy: Mechanism, Management, and Mitigation of Inflammatory Toxicities.

Front. Immunol. 12:693016. doi: 10.3389/fimmu.2021.693016

\section{CAR-T Cell Therapy: Mechanism, Management, and Mitigation of Inflammatory Toxicities}

\author{
Joseph W. Fischer ${ }^{\dagger}$ and Nirjal Bhattarai ${ }^{*}$
}

Division of Cellular and Gene Therapies, Office of Tissues and Advanced Therapies, Center for Biologics Evaluation and Research, U.S. Food and Drug Administration, Silver Spring, MD, United States

Engineered $T$ cell therapies such as chimeric antigen receptor (CAR) expressing $T$ cells (CAR-T cells) have great potential to treat many human diseases; however, inflammatory toxicities associated with these therapies present safety risks and can greatly limit its widespread use. This article briefly reviews our current understanding of mechanisms for inflammatory toxicities during CAR T-cell therapy, current strategies for management and mitigation of these risks and highlights key areas of knowledge gap for future research.

Keywords: CAR-T cells, inflammation, toxicities, CRS, neurotoxicity

\section{INTRODUCTION}

Although CAR-T cell therapy has been in development for more than two decades (1), recent U.S. FDA approval of one BCMA-targeting and several CD19-targeting CAR-T cell therapies for certain relapsed/refractory hematologic malignancies have energized the field (2-8). Many other CAR-T cells targeting various antigens other than CD19 are either in pre-clinical development or currently in clinical trials to treat various human diseases such as cancer, infectious diseases, autoimmune diseases, cardiac diseases etc. $(9,10)$. It is anticipated that the field of CAR-T cell therapy will continuously grow, and new CAR-T cells will be developed to treat previously incurable human diseases.

Based on the data obtained from clinical studies with CD19 CAR-T cell therapies, CAR-T cells can be curative in those patients who respond to the therapy; however, not all patients respond in a similar manner and challenges such as resistance and relapses have been observed $(3,11-14)$. Following infusion, CAR-T cells may fail to proliferate or persist leading to loss of therapeutic response (15). One of the mechanisms that can lead to loss of function is CAR-T cell exhaustion (3, 12). Strategies such as use of checkpoint inhibitors (e.g. anti-PD-1 or anti-PD-L1) to block PD-1 and $\mathrm{PD}-\mathrm{L} 1$ interaction between CAR-T cells and cancer cells are being used in clinical trials to restore CAR-T cell function $(15,16)$. Other strategies to mitigate CAR-T cell exhaustion are also in development (17-19).

Loss or decrease in target antigen expression can also affect efficacy of CAR-T cell therapy. Antigen-null or antigen-dim cancer cells can arise via various mechanisms, which can contribute to resistance to CAR-T therapy (20-23). Experience with CD19 CAR-T cells suggest a significant number of patients can relapse due to loss of CD19 expression (11). Other rare events such as transduction of a tumor cell during manufacturing and subsequent CAR expression in the 
malignant cell resulting in resistance to CAR-T cell therapy has been also observed (24). Thus, numerous mechanisms may contribute to resistance and relapse during CAR-T cell therapy. Therefore, future studies are warranted to better understand mechanisms for resistance and relapses during CAR-T cell therapy and develop novel strategies to overcome these challenges.

CARs are synthetic receptors that are developed to interact with target cells with high specificity. Although CAR-T cells with high specificity are desirable, expression of target antigen in normal cells can lead to unwanted toxicities. For example, CD19targeting CAR T cells effectively kill both CD19+ tumor cells and CD19+ normal B cells resulting in prolonged B cell aplasia (25). Long-term B cell aplasia can be detrimental and can lead to increased risk of infections (25). Expression of target antigen in normal cells can also pose a significant safety risk. In one study, a patient receiving an ERBB2-targeting CAR-T cell therapy died after 5 days, because the CAR-T cells unintentionally targeted lung epithelial cells that expressed low levels of ERBB2 (26). Thus, it is critical to develop strategies to specifically target diseased cells while sparing healthy cells during CAR-T cell therapy (27).

Another hallmark challenge associated with almost all CAR-T cell therapies is development of systemic inflammatory toxicities such as cytokine release syndrome (CRS) and neurotoxicity (28). CRS symptoms can include flu-like symptoms, hypotension, capillary leak, hypoxia, and severe CRS can lead to multi-organ failure. Neurotoxicity symptoms can include headaches, delirium, seizures, and cerebral edema. These symptoms usually occur within the first two weeks, but more severe cases can present within 72 hours (28). In CD19 CAR-T cell therapies, CRS severity was found to vary significantly among patients with up to $46 \%$ of severe cases observed in some studies, which typically requires admittance to the intensive care unit (2-6, 29). These inflammatory toxicities have been observed in almost all CAR-T cell clinical trials, including the FDA-approved CAR$\mathrm{T}$ cell therapies Kymriah, Yescarta, Tecartus, Breyanzi and Abecma (2-7). While new CAR-T cell clinical trials are still facing these challenges, methods to diagnose and better treat inflammatory toxicities during CAR-T cell therapy are also in development $(6,30,31)$.

One of the major challenges for developing effective treatment strategies for inflammatory toxicities during CAR-T cell therapy is poor understanding of mechanisms for heterogenous inflammatory response and factors that contribute to these toxicities. CAR-T cells are also being developed to treat various solid tumors; however, there are several challenges that need to be addressed to improve safety and efficacy of CAR-T cells against solid tumors (32). Furthermore, severity of inflammatory toxicities that may arise following CAR-T cell treatment against solid tumors is poorly understood. Thus, future work to understand mechanisms for inflammatory toxicities and identification of factors that contribute to these toxicities during CAR-T cell therapy against solid and liquid tumors may help in rational design of CAR-T cells that are safer and may also help in developing novel strategies to effectively manage and treat these toxicities.

\section{CURRENT UNDERSTANDING OF MECHANISMS CONTRIBUTING TO INFLAMMATORY TOXICITIES}

Inflammatory toxicities such as CRS and neurotoxicity are associated with the presence of high levels of inflammatory proteins and cytokines such as GM-CSF, IL-6, IL-1 $\beta$, C-reactive protein (CRP), etc. in the serum of patients treated with CAR-T cells $(33,34)$. One of the major sources of these pro-inflammatory cytokines are myeloid cells that are activated during CAR-T cell therapy (35-38). Post-infusion and following target cell recognition, CAR-T cells are activated and secrete various inflammatory factors, such as GM-CSF, which can activate myeloid cells and promote the rapid production and secretion of pro-inflammatory cytokines such as IL- 6 and IL- $1 \beta$ that contribute to inflammatory toxicities $(37,39)$. Since, cytokines like IL- 6 and IL-1 $\beta$ are not primarily produced by CAR-T cells, targeting these cytokines by immunomodulatory agents may not completely prevent CRS and neurotoxicity; however, understanding mechanisms for myeloid cell activation during CAR-T cell therapy may help develop strategies that can prevent or reduce activation of myeloid cells. One such strategy to reduce myeloid activation is through reducing extraneous CAR-T cell activation that can occur from on-target/off-tumor activity (40). Thus, by targeting antigens that are expressed only in diseased cells or by implementing better strategies to reduce on-target/off-tumor activity, unwanted CAR-T cell activation may be reduced, which may help reduce bystander myeloid cell activation.

Toxicity from CAR-T cell therapies can greatly vary, which can be due to multiple factors including patient heterogeneity, baseline tumor burden, CAR-T cell dose, differences in starting material for autologous products or the CAR-T cell manufacturing process (34).Tumor burden can be predictive of toxicities as clinical studies have found that patients with a higher baseline tumor burden or treated with higher number of CAR-T cells have greater incidence of inflammatory toxicities and demonstrate poor survival compared to patients with lower tumor burden $(34,41)$. Other factors, such as patient microbiome, metabolome, or cytokine profile etc. may also modulate CAR-T efficacy and safety and warrant further investigation $(42,43)$.

Manufacturing process can also greatly affect CAR-T cell safety and efficacy (44). For example, selection of CD4 and CD8 positive $\mathrm{T}$ cells following apheresis during manufacturing improved CAR-T cell efficacy, but this process also resulted into CAR-T cells with increased inflammatory toxicities (45). T cell subsets or activation and/or differentiation state of $\mathrm{T}$ cells in the CAR-T cell product can also impact CAR-T cell safety and efficacy. CAR-T cell products containing higher numbers of stem-like memory cells or central memory have demonstrated improved expansion, persistence and efficacy $(46,47)$. Single-cell analysis of various CAR-T cell subsets has revealed different populations of cells contribute to different inflammatory cytokines and effector function (48). Further studies on impact of various $\mathrm{T}$ cell subsets present in the CAR-T cell product on safety and efficacy may help improve safety by allowing for either enrichment or depletion of specific T cell subset. 
CAR-T cell design and choice of co-stimulatory signaling molecules can also impact safety and efficacy (19). Firstgeneration CARs used a single signaling domain, which was found to have poor persistence and low efficacy (49-52). The first-generation CARs were primarily derived from murine antibodies, which may have also contributed to poor persistence and low efficacy. With the addition of a costimulatory domain in the second-generation CARs such as the FDA-approved CAR-T cell products, CAR-T cell efficacy was greatly improved. However, patients treated with these more active CAR-T products also experienced inflammatory toxicities (2-6). Additionally, studies have evaluated correlation between specific domains and susceptibility to inflammatory toxicities. For the five FDA-approved CAR-T cell products, they either contain the CD28 (Yescarta and Tecartus) or 4-1BB (Kymriah, Breyanzi and Abecma) as co-stimulatory signaling domain, correlation between signaling domains and toxicity has remained inconclusive in part due to differences in clinical grading scales and cancer types (19). Due to improvements in inflammatory toxicity management over recent years, it has been challenging to directly compare various studies as more recent studies treat patients earlier or even prophylactically in some cases (6). Thus, it will be important for future clinical studies to compare the safety and efficacy of new emerging CAR-T therapies with other CAR-T cells to better understand effect of various co-stimulatory domains on inflammatory toxicities.

While second generation CAR constructs are used in FDAapproved products and many more are in clinical development, some clinical studies are also assessing third generation CAR constructs that often contain both the CD28 and 4-1BB stimulatory domains (31). Although due to differences between patient population, trial design etc. results from these thirdgeneration CAR-T cells cannot be compared with secondgeneration CAR-T cells, results from early trials have been encouraging and have shown that third-generation CAR-T cells can achieve higher levels of activation without increasing the frequency and severity of inflammatory toxicities $(31,53)$. The increased activation without increased toxicity may be due to the earlier use of immunomodulators, rapid CAR-T exhaustion, differences in baseline tumor burden or other unknown mechanisms that warrant further investigation Data from a multiarm clinical trial using either second-generation or third-generation CAR-T cells may also provide additional insights into this observation. As new CARs are designed and used to treat various diseases, it will be important to better understand how these changes will impact not only their ability to eliminate target cells, but also produce sustained effect and reduce the risk of severe side-effects.

\section{CURRENT STRATEGIES FOR MANAGEMENT OF INFLAMMATORY TOXICITIES AND LIMITATIONS}

Currently, following onset of inflammatory toxicities, treatment strategies focus on reducing overall inflammation by using corticosteroids, or inhibiting inflammatory cytokines, such as IL-6 or IL-1 $\beta$, signaling pathways $(28,54)$. Although, administration of the anti-inflammatory therapies are typically given following onset of CRS; some studies have also used it prophylactically (30). While these treatment strategies are effective in reducing severity of these inflammatory toxicities, they do not prevent their occurrence, nor do patients uniformly respond to these interventions (28). Furthermore, immunosuppressive agents such as corticosteroids have a systemic effect, blunting immune cell responses that can impact therapeutic efficacy. Some studies suggest that the effect of corticosteroids on CAR-T cell function is likely to be minimal $(30,55)$; however, other studies have found it to be inhibitory (56). These differences may be due to the timing, duration and dose of steroids used in these studies. In addition, corticosteroid resistant inflammatory toxicities have been observed during CAR-T cell therapy resulting in multi-organ failure and death (57).

Pro-inflammatory cytokines such as IL-6 and IL-1 $\beta$ are commonly associated with inflammatory toxicities during CAR-T cell therapy and these cytokines are significantly elevated in the blood following CAR-T cell infusion (35). These myeloid-derived cytokines activate other immune cells including themselves (35). Thus, anti-IL- 6 or anti-IL-1 $\beta$ therapy could prevent the positive-feedback loop of myeloid activation and help reduce CRS severity. Currently tocilizumab, an IL-6 receptor antagonist, is the only FDA-approved therapy for treating CAR-T cell-associated CRS (58). Additionally, it has been also used as a preventative treatment, including patients with high tumor burdens, as it does not seem to affect CAR-T cell function and can mitigate the initial severity of CRS $(30,59)$. While tocilizumab has shown promise in resolving CRS severity, it has many limitations. For example, tocilizumab has been found to be less effective in resolving neurotoxicity symptoms $(60,61)$. This is likely because tocilizumab is a large antibody molecule, which is unable to cross the blood brain barrier (BBB) (62). Treatment with tocilizumab can also be clinically ineffective in up to $30 \%$ of patients (58), and tocilizumab-refractory CRS can develop in some patients $(57,63)$. Since tocilizumab targets the IL-6 receptor and not the cytokine, its effect in some patients may be reduced due to the presence of soluble IL- 6 receptor. Further studies need to evaluate the impact of other therapies on CAR-T cell associated inflammatory toxicities, such as siltuximab that binds to the IL-6 cytokine and removes it from circulation (64). Furthermore, preclinical studies have shown that IL-6 deficient mice still develop CRS symptoms when treated with CAR-T cells (65) suggesting targeting IL-6 alone may not be sufficient in all patients and additional therapeutic strategies are needed to treat both CRS and neurotoxicity.

One such inhibitor that may help treat CRS and neurotoxicity when used in in conjunction with IL-6 inhibitors is anakinra. Anakinra is an antibody-based IL-1 receptor (IL-1R) antagonist, which is an FDA-approved drug for treating patients with rheumatoid arthritis and other inflammatory diseases (66). While the use of anakinra for treating CAR-T cell induced toxicities requires additional clinical trials, this therapy may be 
effective in treating both CRS and neurotoxicity $(54,67)$. Previous work has found that anakinra and IL-1 $\beta$ can traverse the $\operatorname{BBB}(33,36,68)$, and in preclinical studies anakinra reduced both CRS and neurotoxicity $(35,36)$. However, similar to tocilizumab, blocking IL- $1 \beta$ signaling by anakinra may not completely treat inflammatory toxicities as IL-1R deficient mice also developed CRS symptoms following CAR-T cell infusion (65). Together, these data suggest that inhibition of inflammatory cytokines such as IL- 6 and IL-1 $\beta$ may not be sufficient to treat inflammatory toxicities in all patients. Furthermore, the effect of these agents on bystander immune responses that also contributes to CAR-T cell efficacy is unknown. Future studies are required to better understand mechanisms contributing to these inflammatory toxicities during CAR-T cell therapy, which may aid in better management of these toxicities. It is also important to note that while current management strategies have several limitations, CAR-T cell therapy has saved many lives. Thus, current toxicity management strategies should continue to be used with CAR-T cell therapy until better management or preventive strategies are identified.

\section{CURRENT STRATEGIES TO PREVENT INFLAMMATORY TOXICITIES DURING CAR-T CELL THERAPY}

In response to the toxicities that accompany CAR-T cell therapy, research has focused on rational CAR design and genetic manipulation of $\mathrm{T}$ cells in order to prevent inflammatory toxicities. New CAR constructs have been designed to contain additional domains to improve CAR-T cell activity (69). These multi-CARs increase the specificity through either requiring recognition of two antigens on the cells surface (bispecific CAR) or requiring the absence of an antigen (inhibitory CAR) (70-72). The increase in specificity can reduce unwanted CAR-T cell activation by reducing on-target/off-tumor activity, which may result into lower levels of inflammatory factors released by CAR-T cells.

Other methods are also in development to increase on-target activity. Expression of chemokine receptors like CCR4, CXCR1, or CXCR2, improved anti-tumor activity by increasing CAR-T cell localization to tumors $(73,74)$. Increased cellular localization to tumors may also help reduce on-target/off-tumor activation of CAR-T cells and peripheral myeloid cells. Additionally, CARs can include molecular switches that can be controlled postinfusion to modulate the level of CAR-T activation (69). While these strategies can help mitigate side-effects by reducing overall activity, they do not directly inhibit the development of inflammatory toxicities.

Use of small molecule inhibitors to prevent inflammatory cytokines signaling may also help reduce severity of inflammatory toxicities in patients. For example, Bruton's tyrosine kinase inhibitor (BTKI) has been shown to reduce production of the inflammatory cytokine IFN-gamma from both CAR-T cells and tumor cells (75). BTKIs are FDAapproved drugs and are frequently used to treat hematological cancers prior to CAR-T cell therapy $(6,76,77)$. One study found that pre-treatment with Ibrutinib (BTKI), was able to effectively reduce CRS severity (78), and this strategy is currently being evaluated in an ongoing clinical trial (NCT03960840). It has been also proposed that BTKIs reduce CRS severity through reducing the expression of inflammatory cytokines, such as IL-6 and GMCSF (75). Although these early findings are promising, the effect of BTKIs on safety and efficacy of CAR-T cell therapies needs to be further studied.

Another small molecule, metyrosine, have been shown to prevent inflammatory toxicities through inhibiting catecholamine production (38). Catecholamines (e.g. dopamine) are secreted by CAR-T cells and act as an endogenous immunomodulator by inducing expression of various cytokines, including IL-6 and IL-8 (79). In an in vivo preclinical study, prophylactic treatment of metyrosine significantly reduced inflammatory cytokine levels without affecting efficacy (38). Metyrosine is an antihypertensive drug used to treat pheochromocytoma (80). Like metyrosine, atrial natriuretic peptide also inhibited catecholamines and reduced CRSassociated cytokine production from CAR-T cells (38). Since catecholamines have previously been linked to neurotoxicities (81), inhibition of catecholamines may help reduce both CRS and neurotoxicity.

Modulation of post-transcriptional processes have been also studied as a potential strategy to reduce inflammatory cytokines during CAR-T cell therapy. JTE-607 (TO-207) is a CPSF3 inhibitor that blocks pre-mRNA processing into mature mRNA and has been shown to reduce the processing and secretion of cytokines from monocytes in vitro (82). This mRNA-processing inhibitor had minimal effects on the release of soluble factors from CAR-T cells, suggesting that it selectively inhibits cytokine production in monocytes (82). An early clinical study found that a single dose of JTE-607 appeared to be welltolerated and reduced the severity of endotoxin-induced inflammation in healthy individuals (83). If this inhibitor can effectively prevent the release of multiple cytokines without effecting the mRNA processing in CAR-T cells and other healthy tissues, it could be more effective than therapies that only target a single cytokine. However, the mechanism of selectively inhibiting only cytokine mRNA processing, while not effecting other cells or transcripts, requires further research. Thus, small molecule drugs can be effective in managing or treating inflammatory toxicities during CAR-T cell therapy. However, these drugs can also affect tumor cells and impact CAR-T cell efficacy, thus, effect of small molecule drugs on tumor cells should be also carefully assessed before using these drugs for treating CAR-T cell toxicities.

Another method that has been in development to modulate inflammatory cytokine expression is through genetic manipulation of CAR-T cells. As IL- 6 and IL- $1 \beta$ are crucial cytokines involved in inflammatory toxicities, CAR-T cells have been engineered to express and secret interleukin-1 receptor (IL1RA), which can sequester free IL-1 $\beta$ in circulation (36). This approach is similar to the strategy of using anakinra, but in this 
strategy, IL-1RA would be expressed prior to IL-1 $\beta$ induction and be continuously released into the blood from CAR-T cells. A similar approach could be used with an IL-6 receptor alone or in combination with IL-1RA. Another study found that knockdown of IL-6 in CAR-T cells reduced IL- 6 production from monocytes, which may be due to reduction in IL-6 positive-feedback loop (39). Thus, these various strategies to design CAR-T cells to prevent IL- 6 and IL-1 $\beta$ signaling may help reduce severity of inflammatory toxicities during CAR-T cell therapy.

Over-expression of CD40 ligand (CD40L) on CAR-T cells increased anti-tumor efficacy through CD40L interactions with CD40 expressed on tumors (84). The over-expression of CD40L also increased expression of IL-1RA resulting into lower IL-1 $\beta$ expression (84). Thus, this strategy of CD40L expression in CAR-T cells may help reduce inflammatory toxicities; however, CD40L also interacts with CD40 on antigen presenting cells, which may lead to increased activation of myeloid cells (35). Thus, future studies should carefully evaluate the effect of overexpression of CD40L on CAR- T cell safety and efficacy.

While targeting inflammatory cytokines such as IL- 6 or IL-1 $\beta$ may help reduce severity of inflammatory toxicities during CAR$\mathrm{T}$ cell therapy, it does not prevent the onset of these toxicities. Since these inflammatory cytokines are not primarily produced by CAR-T cells (85), targeting CAR-T cell factors that contribute to myeloid cell activation may be more effective in preventing onset of these toxicities. GM-CSF is one of those promising factors, which is secreted by CAR T-cells following activation and activates myeloid cells but does not appear to contribute to CAR-T cell function $(37,65,86)$. In vitro inactivation of GMCSF in CAR-T cells reduced myeloid cell-derived inflammatory cytokines (37), and in some studies GM-CSF inhibition reduced expression of some CRS associated cytokines, such as IL-6, but not IL-1 $\beta(37,65,86-88)$. However, in animal studies, GM-CSF neutralization did not completely prevent inflammatory toxicities (86). As the mechanisms for inflammatory toxicities are likely to be very complex, it is anticipated that additional factors released by CAR-T cells contribute to these inflammatory toxicities. Thus, future studies are warranted to identify these novel inflammatory factors released by CAR-T cells to help rationally design CAR-T cells that are less toxic.

Current strategies to rationally improve CAR-T cells have primarily focused on improving its efficacy (10). One of such modifications is deletion of the NR4A transcription factors to reduce CAR-T cell exhaustion and improve efficacy (18). Although, NR4A deletion improved CAR-T cell potency in preclinical animal studies, GM-CSF was significantly upregulated over 20 -fold in the NR4A knockout CAR-T cells. This suggests that while deletion of NR4A may improve CAR-T cell function, it may also significantly increase myeloid cell activation and inflammatory toxicities. Thus, multiple genetic manipulations may be required to improve overall safety and efficacy of CAR-T cells. Genome editing tools have greatly

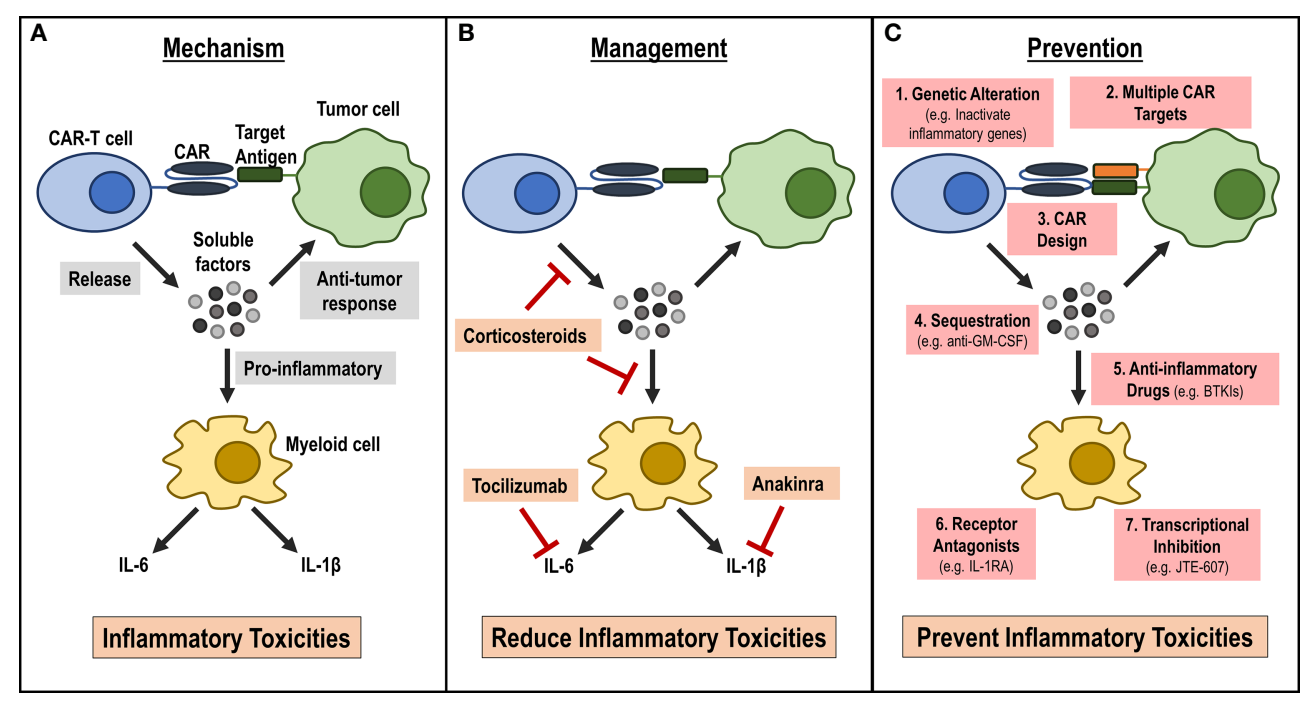

FIGURE 1 | Current understanding of mechanism for inflammatory toxicities during CAR-T cell therapy, current management strategies and future strategies for prevention. (A) Mechanism: Activated CAR-T cells release soluble factors upon CAR engagement with target antigen. These soluble factors can aid in the anti-tumor response, or they can activate bystander myeloid cells. Activated myeloid cells secrete inflammatory cytokines, such as IL-6 and IL-1 $\beta$, that lead to the inflammatory toxicities observed in patients infused with CAR-T cells. (B) Management: Current management strategies focus on reducing inflammatory cytokines or cytokine signaling pathways by either using anti-inflammatory drugs, such as corticosteroids that may reduce inflammatory cytokines release by CAR-T cells or myeloid cells or by targeting specific cytokine receptors, such as IL-6R by tocilizumab and IL-1R by anakinra. (C) Prevention: New approaches that focus on preventing the onset of CRS. These strategies include: 1) modifying CAR-T cells during manufacturing such as genetic alterations to inactivate inflammatory genes, 2) designing CARs with novel domains that are less inflammatory, 3) targeting multiple antigens on tumors to reduce on-target off-tumor activation, 4) inhibiting pro-inflammatory CAR-T cell factors using antibodies (e.g. anti-GM-CSF), or inhibiting myeloid cell activation by 5) using small molecule inhibitors (e.g. BTKI), 6) expressing inflammatory cytokine receptor (e.g. IL-1R) on CAR-T cells or 7) using myeloid cell-specific transcriptional Inhibitor (e.g. JTE-607). 
improved in recent years and can effectively knock-out, modify, and insert genes within primary cells. With these improvements, it has become possible to incorporate a variety of modifications to $\mathrm{T}$ cells during CAR-T cell manufacturing. As new genetic modifications are introduced to improve CAR-T cell safety and efficacy, the effect of these modifications should be carefully evaluated, and a comprehensive risk assessment should be performed prior to initiation of clinical studies.

Other immune cells such as natural killer (NK) cells that express CAR (CAR-NK) are also in development (89). In a phase I clinical study, anti-CD19 CAR-NK cells demonstrated a potent anti-tumor response without causing any severe inflammatory toxicities (90). Although, additional studies will be required to further corroborate safety and efficacy of CAR-NK cells, these early data suggest that CAR-NK cells may be safer than CAR-T cells. Furthermore, future studies of CAR-NK cells and characterization of mechanism contributing to lower inflammatory toxicities during CAR-NK cell therapy may also provide insights into mechanism for inflammatory toxicities during CAR-T cell therapy and aid in rationally designing CAR-T cells that are safer and effective.

\section{CONCLUSIONS AND FUTURE PERSPECTIVES}

As CAR-T cell therapies are being developed to treat a wide variety of human diseases, it is critical to understand inflammatory toxicities associated with these therapies and develop strategies to effectively manage or prevent these adverse events (Figure 1). While current treatment strategies help reduce the severity and duration of inflammatory toxicities, there are several limitations of these current strategies. Additionally, current management strategies can have heterogeneous response in patients, can be toxic and are

\section{REFERENCES}

1. Eshhar Z, Waks T, Gross G, Schindler DG. Specific Activation and Targeting of Cytotoxic Lymphocytes Through Chimeric Single Chains Consisting of Antibody-Binding Domains and the Gamma or Zeta Subunits of the Immunoglobulin and T-Cell Receptors. Proc Natl Acad Sci USA (1993) 90:720-4. doi: 10.1073/pnas.90.2.720

2. Maude SL, Laetsch TW, Buechner J, Rives S, Boyer M, Bittencourt H, et al. Tisagenlecleucel in Children and Young Adults With B-Cell Lymphoblastic Leukemia. N Engl J Med (2018) 378:439-48. doi: 10.1056/NEJMoa1709866

3. Schuster SJ, Bishop MR, Tam CS, Waller EK, Borchmann P, McGuirk JP, et al. Tisagenlecleucel in Adult Relapsed or Refractory Diffuse Large B-Cell Lymphoma. N Engl J Med (2019) 380:45-56. doi: 10.1056/NEJMoa1804980

4. Neelapu SS, Locke FL, Bartlett NL, Lekakis LJ, Miklos DB, Jacobson CA, et al. Axicabtagene Ciloleucel CAR T-Cell Therapy in Refractory Large B-Cell Lymphoma. N Engl J Med (2017) 377:2531-44. doi: 10.1056/NEJMoa1707447

5. Locke FL, Ghobadi A, Jacobson CA, Miklos DB, Lekakis LJ, Oluwole OO, et al. Long-Term Safety and Activity of Axicabtagene Ciloleucel in Refractory Large BCell Lymphoma (ZUMA-1): A Single-Arm, Multicentre, Phase 1-2 Trial. Lancet Oncol (2019) 20:31-42. doi: 10.1016/S1470-2045(18)30864-7

6. Wang M, et al. KTE-X19 CAR T-Cell Therapy in Relapsed or Refractory MantleCell Lymphoma. N Engl J Med (2020) 382:1331-42. doi: 10.1056/NEJMoa1914347

7. Abramson JS, Munoz J, Goy A, Locke FL, Jacobson CA, Hill BT, et al. Lisocabtagene Maraleucel for Patients With Relapsed or Refractory Large expensive. Furthermore, they do not target the underlying cause of inflammatory toxicities during CAR-T cell therapy. Understanding mechanisms contributing to inflammatory toxicities during CAR-T cell therapies will help to greatly improve safety of these therapies by helping develop less toxic CAR-T cells during manufacturing and by developing better treatment strategies for patients who develop these toxicities (Figure 1). As the CAR-T cell field grows, it is anticipated that the CAR-T cell design and manufacturing process will be more complex. Thus, it is critical that future studies address these underlying challenges in an urgent manner so that the benefit of this effective therapy in treating numerous human diseases can reach to widespread population.

\section{AUTHOR CONTRIBUTIONS}

All authors contributed to the article and approved the submitted version.

\section{ACKNOWLEDGMENTS}

We thank Winston Colon-Moran and Drs. Alan Baer, Pankaj Mandal, Bharat Joshi and Raj Puri for critical reading of this manuscript and helpful comments. We also thank Dr. Catherine Fischer for her input on figure design. This work was supported by the Intramural Research Program of the Center for Biologics Evaluation and Research (CBER), U.S. Food and Drug Administration. This project was also supported in part by Dr. Joseph Fischer's appointment to the Research Participation Program at CBER administered by the Oak Ridge Institute for Science and Education through the US Department of Energy and U.S. Food and Drug Administration.

B-Cell Lymphomas (TRANSCEND NHL 001): A Multicentre Seamless Design Study. Lancet (2020) 396:839-52. doi: 10.1016/S0140-6736(20)31366-0

8. FDA. FDA Approves First Cell-Based Gene Therapy for Adult Patients With Multiple Myeloma. (2021). Available at: https://www.fda.gov/news-events/ press-announcements/fda-approves-first-cell-based-gene-therapy-adultpatients-multiple-myeloma.

9. Maldini CR, Ellis GI, Riley JL. CAR T Cells for Infection, Autoimmunity and Allotransplantation. Nat Rev Immunol (2018) 18:605-16. doi: 10.1038/ s41577-018-0042-2

10. Tian Y, Li Y, Shao Y, Zhang Y. Gene Modification Strategies for NextGeneration CAR T Cells Against Solid Cancers. J Hematol Oncol (2020) 13:54. doi: 10.1186/s13045-020-00890-6

11. Fousek K, Watanabe J, Joseph SK, George A, An X, Byrd TT, et al. CAR T-Cells That Target Acute B-Lineage Leukemia Irrespective of CD19 Expression. Leukemia (2020) 35:75-89. doi: 10.1038/s41375-020-0792-2

12. Maude SL, Barrett DM, Rheingold SR, Aplenc R, Teachey DT, Callahan C, et al. Efficacy of Humanized CD19-Targeted Chimeric Antigen Receptor (CAR)-Modified T Cells in Children and Young Adults With Relapsed/Refractory Acute Lymphoblastic Leukemia. Blood (2016) 128:217-7. doi: 10.1182/blood.V128.22.217.217

13. Shah NN, Lee DW, Yates B, Yuan CM, Shalabi H, Martin S, et al. Long-Term Follow-Up of CD19-CAR T-Cell Therapy in Children and Young Adults With B-ALL. J Clin Oncol (2021) 39:1650-9. doi: 10.1200/JCO.20.02262

14. Kalos M, Levine BL, Porter DL, Katz S, Grupp SA, Bagg A, et al. T Cells With Chimeric Antigen Receptors Have Potent Antitumor Effects and can Establish 
Memory in Patients With Advanced Leukemia. Sci Transl Med (2011) 3:95ra73. doi: 10.1126/scitranslmed.3002842

15. Jafarzadeh L, Masoumi E, Fallah-Mehrjardi K, Mirzaei HR, Hadjati J. Prolonged Persistence of Chimeric Antigen Receptor (CAR) T Cell in Adoptive Cancer Immunotherapy: Challenges and Ways Forward. Front Immunol (2020) 11:702. doi: 10.3389/fimmu.2020.00702

16. McGowan E, Lin Q, Ma G, Yin H, Chen S, Lin Y, et al. PD-1 Disrupted CAR-T Cells in the Treatment of Solid Tumors: Promises and Challenges. BioMed Pharmacother (2020) 121:109625. doi: 10.1016/j.biopha.2019.109625

17. Lynn RC, Weber EW, Sotillo E, Gennert D, Xu P, Good Z, et al. C-Jun Overexpression in CAR T Cells Induces Exhaustion Resistance. Nature (2019) 576:293-300. doi: 10.1038/s41586-019-1805-z

18. Chen J, Lopez-Moyado IF, Seo H, Lio CJ, Hempleman LJ, Sekiya T, et al. NR4A Transcription Factors Limit CAR T Cell Function in Solid Tumours. Nature (2019) 567:530-4. doi: 10.1038/s41586-019-0985-x

19. Weinkove R, George P, Dasyam N, McLellan AD. Selecting Costimulatory Domains for Chimeric Antigen Receptors: Functional and Clinical Considerations. Clin Transl Immunol (2019) 8:e1049. doi: 10.1002/cti2.1049

20. Orlando EJ, Han X, Tribouley C, Wood PA, Leary RJ, Riester M, et al. Genetic Mechanisms of Target Antigen Loss in CAR19 Therapy of Acute Lymphoblastic Leukemia. Nat Med (2018) 24:1504-6. doi: 10.1038/s41591018-0146-Z

21. Fischer J, Paret C, El Malki K, Alt F, Wingerter A, Neu MA, et al. CD19 Isoforms Enabling Resistance to CART-19 Immunotherapy are Expressed in B-ALL Patients at Initial Diagnosis. J Immunother (2017) 40:187-95. doi: 10.1097/CJI.0000000000000169

22. Sotillo E, Barrett DM, Black KL, Bagashev A, Oldridge D, Wu G, et al. Convergence of Acquired Mutations and Alternative Splicing of CD19 Enables Resistance to CART-19 Immunotherapy. Cancer Discovery (2015) 5:1282-95. doi: 10.1158/2159-8290.CD-15-1020

23. Gardner R, Wu D, Cherian S, Fang M, Hanafi LA, Finney O, et al. Acquisition of a CD19-Negative Myeloid Phenotype Allows Immune Escape of MLLRearranged B-ALL From CD19 CAR-T-Cell Therapy. Blood (2016) 127:240610. doi: 10.1182/blood-2015-08-665547

24. Ruella M, Xu J, Barrett DM, Fraietta JA, Reich TJ, Ambrose DE, et al. Induction of Resistance to Chimeric Antigen Receptor T Cell Therapy by Transduction of a Single Leukemic B Cell. Nat Med (2018) 24:1499-503. doi: 10.1038/s41591-018-0201-9

25. Maude SL, Frey N, Shaw PA, Aplenc R, Barrett DM, Bunin NJ, et al. Chimeric Antigen Receptor T Cells for Sustained Remissions in Leukemia. N Engl J Med (2014) 371:1507-17. doi: 10.1056/NEJMoa1407222

26. Morgan RA, Yang JC, Kitano M, Dudley ME, Laurencot CM, Rosenberg SA. Case Report of a Serious Adverse Event Following the Administration of $\mathrm{T}$ Cells Transduced With a Chimeric Antigen Receptor Recognizing ERBB2. Mol Ther (2010) 18:843-51. doi: 10.1038/mt.2010.24

27. Huang R, Li X, He Y, Zhu W, Gao L, Liu Y, et al. Recent Advances in CAR-T Cell Engineering. J Hematol Oncol (2020) 13:86. doi: 10.1186/s13045-02000910-5

28. Rivera AM, May S, Lei M, Qualls S, Bushey K, Rubin DB, et al. CAR T-CellAssociated Neurotoxicity: Current Management and Emerging Treatment Strategies. Crit Care Nurs Q (2020) 43:191-204. doi: 10.1097/ CNQ.0000000000000302

29. Fitzgerald JC, Weiss SL, Maude SL, Barrett DM, Lacey SF, Melenhorst JJ, et al. Cytokine Release Syndrome After Chimeric Antigen Receptor T Cell Therapy for Acute Lymphoblastic Leukemia. Crit Care Med (2017) 45:e124-31. doi: 10.1097/CCM.0000000000002053

30. Gardner RA, Ceppi F, Rivers J, Annesley C, Summers C, Taraseviciute A, et al. Preemptive Mitigation of CD19 CAR T-Cell Cytokine Release Syndrome Without Attenuation of Antileukemic Efficacy. Blood (2019) 134:2149-58. doi: 10.1182/blood.2019001463

31. Enblad G, Karlsson H, Gammelgard G, Wenthe J, Lovgren T, Amini RM, et al. A Phase I/Iia Trial Using CD19-Targeted Third-Generation CAR T Cells for Lymphoma and Leukemia. Clin Cancer Res (2018) 24:6185-94. doi: 10.1158/ 1078-0432.CCR-18-0426

32. Marofi F, Marofi F, Motavalli R, Safonov VA, Thangavelu L, Yumashev AV, Alexander M, et al. CAR T Cells in Solid Tumors: Challenges and Opportunities. Stem Cell Res Ther (2021) 12:81. doi: 10.1186/s13287-020-02128-1
33. Luo H, Wang N, Huang L, Zhou X, Jin J, Li C, et al. Inflammatory Signatures for Quick Diagnosis of Life-Threatening Infection During the CAR T-Cell Therapy. J Immunother Cancer (2019) 7:271. doi: 10.1186/s40425-019-0767-x

34. Hay KA, Hanafi LA, Li D, Gust J, Liles WC, Wurfel MM, et al. Kinetics and Biomarkers of Severe Cytokine Release Syndrome After CD19 Chimeric Antigen Receptor-Modified T-Cell Therapy. Blood (2017) 130:2295-306. doi: 10.1182/blood-2017-06-793141

35. Norelli M, Camisa B, Barbiera G, Falcone L, Purevdorj A, Genua M, et al. Monocyte-Derived IL-1 and IL-6 Are Differentially Required for CytokineRelease Syndrome and Neurotoxicity Due to CAR T Cells. Nat Med (2018) 24:739-48. doi: 10.1038/s41591-018-0036-4

36. Giavridis T, van der Stegen SJC, Eyquem J, Hamieh M, Piersigilli A, Sadelain M. CAR T Cell-Induced Cytokine Release Syndrome is Mediated by Macrophages and Abated by IL-1 Blockade. Nat Med (2018) 24:731-8. doi: 10.1038/s41591-018-0041-7

37. Sachdeva M, Duchateau P, Depil S, Poirot L, Valton J. GranulocyteMacrophage Colony-Stimulating Factor Inactivation in CAR T-Cells Prevents Monocyte-Dependent Release of Key Cytokine Release Syndrome Mediators. J Biol Chem (2019) 294:5430-7. doi: 10.1074/jbc.AC119.007558

38. Staedtke V, Bai RY, Kim K, Darvas M, Davila ML, Riggins GJ, et al. Disruption of a Self-Amplifying Catecholamine Loop Reduces Cytokine Release Syndrome. Nature (2018) 564:273-7. doi: 10.1038/s41586-018-0774-y

39. Kang L, Tang X, Zhang J, Li M, Xu N, Qi W, et al. Interleukin-6-Knockdown of Chimeric Antigen Receptor-Modified T Cells Significantly Reduces IL-6 Release From Monocytes. Exp Hematol Oncol (2020) 9:11. doi: 10.1186/ s40164-020-00166-2

40. Pennell CA, Barnum JL, McDonald-Hyman CS, Panoskaltsis-Mortari A, Riddle MJ, Xiong Z, et al. Human CD19-Targeted Mouse T Cells Induce B Cell Aplasia and Toxicity in Human CD19 Transgenic Mice. Mol Ther (2018) 26:1423-34. doi: 10.1016/j.ymthe.2018.04.006

41. Park JH, Riviere I, Gonen M, Wang X, Senechal B, Curran KJ, et al. LongTerm Follow-Up of CD19 CAR Therapy in Acute Lymphoblastic Leukemia. N Engl J Med (2018) 378:449-59. doi: 10.1056/NEJMoa1709919

42. Dougan M, Luoma AM, Dougan SK, Wucherpfennig KW. Understanding and Treating the Inflammatory Adverse Events of Cancer Immunotherapy. Cell (2021) 184:1575-88. doi: 10.1016/j.cell.2021.02.011

43. Hong R, Hu Y, Huang H. Biomarkers for Chimeric Antigen Receptor T Cell Therapy in Acute Lymphoblastic Leukemia: Prospects for Personalized Management and Prognostic Prediction. Front Immunol (2021) 12:627764. doi: 10.3389/fimmu.2021.627764

44. Vormittag P, Gunn R, Ghorashian S, Veraitch FS. A Guide to Manufacturing CAR T Cell Therapies. Curr Opin Biotechnol (2018) 53:164-81. doi: 10.1016/ j.copbio.2018.01.025

45. Shah NN, Highfill SL, Shalabi H, Yates B, Jin J, Wolters PL, et al. CD4/CD8 TCell Selection Affects Chimeric Antigen Receptor (CAR) T-Cell Potency and Toxicity: Updated Results From a Phase I Anti-CD22 CAR T-Cell Trial. J Clin Oncol (2020) 38:1938-50. doi: 10.1200/JCO.19.03279

46. Wang X, Popplewell LL, Wagner JR, Naranjo A, Blanchard MS, Mott MR, et al. Phase 1 Studies of Central Memory-Derived CD19 CAR T-Cell Therapy Following Autologous HSCT in Patients With B-Cell NHL. Blood (2016) 127:2980-90. doi: 10.1182/blood-2015-12-686725

47. Fraietta JA, Lacey SF, Orlando EJ, Pruteanu-Malinici I, Gohil M, Lundh S, et al. Determinants of Response and Resistance to CD19 Chimeric Antigen Receptor (CAR) T Cell Therapy of Chronic Lymphocytic Leukemia. Nat Med (2018) 24:563-71. doi: 10.1038/s41591-018-0010-1

48. Xhangolli I, Dura B, Lee G, Kim D, Xiao Y, Fan R. Single-Cell Analysis of CAR-T Cell Activation Reveals a Mixed TH1/TH2 Response Independent of Differentiation. Genomics Proteomics Bioinf (2019) 17:129-39. doi: 10.1016/ j.gpb.2019.03.002

49. Till BG, Jensen MC, Wang J, Chen EY, Wood BL, Greisman HA, et al. Adoptive Immunotherapy for Indolent Non-Hodgkin Lymphoma and Mantle Cell Lymphoma Using Genetically Modified Autologous CD20-Specific T Cells. Blood (2008) 112:2261-71. doi: 10.1182/blood-2007-12-128843

50. Lamers CH, van Elzakker P, Langeveld SC, Sleijfer S, Gratama JW. Process Validation and Clinical Evaluation of a Protocol to Generate Gene-Modified T Lymphocytes for Imunogene Therapy for Metastatic Renal Cell Carcinoma: GMP-Controlled Transduction and Expansion of Patient's T Lymphocytes 
Using a Carboxy Anhydrase IX-Specific Scfv Transgene. Cytotherapy (2006) 8:542-53. doi: 10.1080/14653240601056396

51. Kershaw MH, Westwood JA, Parker LL, Wang G, Eshhar Z, Mavroukakis SA, et al. A Phase I Study on Adoptive Immunotherapy Using Gene-Modified T Cells for Ovarian Cancer. Clin Cancer Res (2006) 12:6106-15. doi: 10.1158/ 1078-0432.CCR-06-1183

52. Jensen MC, Popplewell L, Cooper LJ, DiGiusto D, Kalos M, Ostberg JR, et al. Antitransgene Rejection Responses Contribute to Attenuated Persistence of Adoptively Transferred CD20/CD19-Specific Chimeric Antigen Receptor Redirected T Cells in Humans. Biol Blood Marrow Transplant (2010) 16:1245-56. doi: 10.1016/j.bbmt.2010.03.014

53. Schubert M-L, Schmitt A, Neuber B, Hückelhoven-Krauss A, Kunz A, Wang L, et al. Third-Generation CAR T Cells Targeting CD19 are Associated With an Excellent Safety Profile and Might Improve Persistence of CAR T Cells in Treated Patients. Blood (2019) 134:51-1. doi: 10.1182/blood-2019-125423

54. Strati P, Ahmed S, Kebriaei P, Nastoupil LJ, Claussen CM, Watson G, et al. Clinical Efficacy of Anakinra to Mitigate CAR T-Cell Therapy-Associated Toxicity in Large B-Cell Lymphoma. Blood Adv (2020) 4:3123-7. doi: 10.1182/ bloodadvances.2020002328

55. Liu S, Deng B, Yin Z, Pan J, Lin Y, Ling Z, et al. Corticosteroids do Not Influence the Efficacy and Kinetics of CAR-T Cells for B-Cell Acute Lymphoblastic Leukemia. Blood Cancer J (2020) 10:15. doi: 10.1038/s41408020-0280-y

56. Davila ML, Riviere I, Wang X, Bartido S, Park J, Curran K, et al. Efficacy and Toxicity Management of 19-28z CAR T Cell Therapy in B Cell Acute Lymphoblastic Leukemia. Sci Transl Med (2014) 6:224ra225. doi: 10.1126/ scitranslmed. 3008226

57. Turtle CJ, Hanafi LA, Berger C, Gooley TA, Cherian S, Hudecek M, et al. CD19 CAR-T Cells of Defined CD4+:CD8+ Composition in Adult B Cell ALL Patients. J Clin Invest (2016) 126:2123-38. doi: 10.1172/JCI85309

58. Le RQ, Li L, Yuan W, Shord SS, Nie L, Habtemariam BA, et al. FDA Approval Summary: Tocilizumab for Treatment of Chimeric Antigen Receptor T CellInduced Severe or Life-Threatening Cytokine Release Syndrome. Oncologist (2018) 23:943-7. doi: 10.1634/theoncologist.2018-0028

59. Kadauke S, Myers RM, Li Y, Aplenc R, Baniewicz D, Barrett DM, et al. RiskAdapted Preemptive Tocilizumab to Prevent Severe Cytokine Release Syndrome After CTL019 for Pediatric B-Cell Acute Lymphoblastic Leukemia: A Prospective Clinical Trial. J Clin Oncol (2021) 39:920-30. doi: 10.1200/JCO.20.02477

60. Kotch C, Barrett D, Teachey DT. Tocilizumab for the Treatment of Chimeric Antigen Receptor T Cell-Induced Cytokine Release Syndrome. Expert Rev Clin Immunol (2019) 15:813-22. doi: 10.1080/1744666X.2019.1629904

61. Santomasso BD, Park JH, Salloum D, Riviere I, Flynn J, Mead E, et al. Clinical and Biological Correlates of Neurotoxicity Associated With CAR T-Cell Therapy in Patients With B-Cell Acute Lymphoblastic Leukemia. Cancer Discovery (2018) 8:958-71. doi: 10.1158/2159-8290.CD-17-1319

62. Nellan A, McCully CML, Cruz Garcia R, Jayaprakash N, Widemann BC, Lee DW, et al. Improved CNS Exposure to Tocilizumab After Cerebrospinal Fluid Compared to Intravenous Administration in Rhesus Macaques. Blood (2018) 132:662-6. doi: 10.1182/blood-2018-05-846428

63. Ishii K, Shalabi H, Yates B, Delbrook C, Mackall CL, Fry TJ, et al. Tocilizumab-Refractory Cytokine Release Syndrome (CRS) Triggered by Chimeric Antigen Receptor (CAR)-Transduced T Cells may Have Distinct Cytokine Profiles Compared to Typical CRS. Blood (2016) 128:3358-8. doi: 10.1182/blood.V128.22.3358.3358

64. Chen F, Teachey DT, Pequignot E, Frey N, Porter D, Maude SL, et al. Measuring IL-6 and Sil-6R in Serum From Patients Treated With Tocilizumab and/or Siltuximab Following CAR T Cell Therapy. J Immunol Methods (2016) 434:1-8. doi: 10.1016/j.jim.2016.03.005

65. Sentman ML, Murad JM, Cook WJ, Wu MR, Reder J, Baumeister SH, et al. Mechanisms of Acute Toxicity in NKG2D Chimeric Antigen Receptor T Cell-Treated Mice. J Immunol (2016) 197:4674-85. doi: 10.4049/ jimmunol.1600769

66. Cavalli G, Dinarello CA. Anakinra Therapy for non-Cancer Inflammatory Diseases. Front Pharmacol (2018) 9:1157. doi: 10.3389/fphar.2018.01157

67. Jatiani SS, Aleman A, Madduri D, Chari A, Cho HJ, Richard S, et al. Myeloma Car-T Crs Management With Il-1r Antagonist Anakinra. Clin Lymphoma Myeloma Leuk (2020) 20:632-6. doi: 10.1016/j.clml.2020.04.020
68. Ogungbenro K, Hulme S, Rothwell N, Hopkins S, Tyrrell P, Galea J, et al. Study Design and Population Pharmacokinetic Analysis of a Phase II Dose-Ranging Study of Interleukin-1 Receptor Antagonist. J Pharmacokinet Pharmacodyn (2016) 43:1-12. doi: 10.1007/s10928-015-9450-0

69. Liu D, Zhao J, Song Y. Engineering Switchable and Programmable Universal Cars for CAR T Therapy. J Hematol Oncol (2019) 12:69. doi: 10.1186/s13045-019-0763-0

70. Amrolia PJ, Wynn R, Hough RE, Vora A, Bonney D, Veys P, et al. Phase I Study of AUTO3, a Bicistronic Chimeric Antigen Receptor (CAR) T-Cell Therapy Targeting CD19 and CD22, in Pediatric Patients With Relapsed/Refractory BCell Acute Lymphoblastic Leukemia (R/R B-ALL): Amelia Study. Blood (2019) 134:2620-0. doi: 10.1182/blood-2019-123424

71. Qin H, Nguyen SM, Ramakrishna S, Tarun S, Yang L, Verdini NP, et al. Novel CD19/CD22 Bicistronic Chimeric Antigen Receptors Outperform Single or Bivalent Cars in Eradicating CD19+CD22+, CD19-, and CD22- Pre-B Leukemia. Blood (2017) 130:810-0. doi: 10.1182/blood.V130.Suppl_1.810.810

72. Fedorov VD, Themeli M, Sadelain M. PD-1- and CTLA-4-Based Inhibitory Chimeric Antigen Receptors (Icars) Divert Off-Target Immunotherapy Responses. Sci Transl Med (2013) 5:215ra172. doi: 10.1126/scitranslmed.3006597

73. Di Stasi A, De Angelis B, Rooney CM, Zhang L, Mahendravada A, Foster AE, et al. T Lymphocytes Coexpressing CCR4 and a Chimeric Antigen Receptor Targeting CD30 Have Improved Homing and Antitumor Activity in a Hodgkin Tumor Model. Blood (2009) 113:6392-402. doi: 10.1182/blood-2009-03-209650

74. Jin L, Tao H, Karachi A, Long Y, Hou AY, Na M, et al. CXCR1- or CXCR2Modified CAR T Cells Co-Opt IL-8 for Maximal Antitumor Efficacy in Solid Tumors. Nat Commun (2019) 10:4016. doi: 10.1038/s41467-019-11869-4

75. Ruella M, Kenderian SS, Shestova O, Klichinsky M, Melenhorst JJ, Wasik MA, et al. Kinase Inhibitor Ibrutinib to Prevent Cytokine-Release Syndrome After Anti-CD19 Chimeric Antigen Receptor T Cells for B-Cell Neoplasms. Leukemia (2017) 31:246-8. doi: 10.1038/leu.2016.262

76. Wang ML, Rule S, Martin P, Goy A, Auer R, Kahl BS, et al. Targeting BTK With Ibrutinib in Relapsed or Refractory Mantle-Cell Lymphoma. N Engl J Med (2013) 369:507-16. doi: 10.1056/NEJMoa1306220

77. Byrd JC, Furman RR, Coutre SE, Flinn IW, Burger JA, Blum KA, et al. Targeting BTK With Ibrutinib in Relapsed Chronic Lymphocytic Leukemia. N Engl J Med (2013) 369:32-42. doi: 10.1056/NEJMoa1215637

78. Gauthier J, Hirayama AV, Hay KA, Li D, Lymp J, Sheih A, et al. Comparison of Efficacy and Toxicity of CD19-Specific Chimeric Antigen Receptor T-Cells Alone or in Combination With Ibrutinib for Relapsed and/or Refractory CLL. Blood (2018) 132:299-9. doi: 10.1182/blood-2018-99-111061

79. Torres KC, Antonelli LR, Souza AL, Teixeira MM, Dutra WO, Gollob KJ. Norepinephrine, Dopamine and Dexamethasone Modulate Discrete Leukocyte Subpopulations and Cytokine Profiles From Human PBMC. J Neuroimmunol (2005) 166:144-57. doi: 10.1016/j.jneuroim.2005.06.006

80. Naruse M, Satoh F, Tanabe A, Okamoto T, Ichihara A, Tsuiki M, et al. Efficacy and Safety of Metyrosine in Pheochromocytoma/Paraganglioma: A Multi-Center Trial in Japan. Endocr J (2018) 65:359-71. doi: 10.1507/ endocrj.EJ17-0276

81. Dina OA, Khasar SG, Alessandri-Haber N, Bogen O, Chen X, Green PG, et al. Neurotoxic Catecholamine Metabolite in Nociceptors Contributes to Painful Peripheral Neuropathy. Eur J Neurosci (2008) 28:1180-90. doi: 10.1111/ j.1460-9568.2008.06425.x

82. Futami M, Suzuki K, Kato S, Ohmae S, Tahara Y, Nojima M, et al. The Novel Multi-Cytokine Inhibitor to-207 Specifically Inhibits Pro-Inflammatory Cytokine Secretion in Monocytes Without Affecting the Killing Ability of CAR T Cells. PloS One (2020) 15:e0231896. doi: 10.1371/journal.pone.0231896

83. Borozdenkova S, Mant TG, Allen E, Pu K, Hoshino S, Jurcevic S, et al. Effects of a Cytokine Inhibitor, JTE-607, on the Response to Endotoxin in Healthy Human Volunteers. Int Immunopharmacol (2011) 11:1837-43. doi: 10.1016/ j.intimp.2011.07.013

84. Kuhn NF, Purdon TJ, van Leeuwen DG, Lopez AV, Curran KJ, Daniyan AF, et al. CD40 Ligand-Modified Chimeric Antigen Receptor T Cells Enhance Antitumor Function by Eliciting an Endogenous Antitumor Response. Cancer Cell (2019) 35:473-88.e476. doi: 10.1016/j.ccell.2019.02.006

85. Barrett DM, Singh N, Hofmann TJ, Gershenson Z, Grupp SA. Interleukin 6 is Not Made by Chimeric Antigen Receptor T Cells and Does Not Impact Their Function. Blood (2016) 128:654-4. doi: 10.1182/blood.V128.22.654.654

86. Sterner RM, Sakemura R, Cox MJ, Yang N, Khadka RH, Forsman CL, et al. GM-CSF Inhibition Reduces Cytokine Release Syndrome and 
Neuroinflammation But Enhances CAR-T Cell Function in Xenografts. Blood (2019) 133:697-709. doi: 10.1182/blood-2018-10-881722

87. Cook AD, Braine EL, Hamilton JA. Stimulus-Dependent Requirement for Granulocyte-Macrophage Colony-Stimulating Factor in Inflammation. J Immunol (2004) 173:4643-51. doi: 10.4049/jimmunol.173.7.4643

88. Lotfi N, Zhang GX, Esmaeil N, Rostami A. Evaluation of the Effect of GM-CSF Blocking on the Phenotype and Function of Human Monocytes. Sci Rep (2020) 10:1567. doi: 10.1038/s41598-020-58131-2

89. Basar R, Daher M, Rezvani K. Next-Generation Cell Therapies: The Emerging Role of CAR-NK Cells. Hematol Am Soc Hematol Educ Program (2020) 2020:570-8. doi: 10.1182/hematology.2020002547

90. Liu E, Marin D, Banerjee P, Macapinlac HA, Thompson P, Basar R, et al. Use of CAR-Transduced Natural Killer Cells in CD19-Positive
Lymphoid Tumors. N Engl J Med (2020) 382:545-53. doi: 10.1056/ NEJMoa 1910607

Conflict of Interest: The authors declare that the research was conducted in the absence of any commercial or financial relationships that could be construed as a potential conflict of interest.

Copyright $\odot 2021$ Fischer and Bhattarai. This is an open-access article distributed under the terms of the Creative Commons Attribution License (CC BY). The use, distribution or reproduction in other forums is permitted, provided the original author(s) and the copyright owner(s) are credited and that the original publication in this journal is cited, in accordance with accepted academic practice. No use, distribution or reproduction is permitted which does not comply with these terms. 